\title{
P12 THE BUTTERFLY PROJECT
}

Debbie Brownlee, ${ }^{1}$ Audra Cook, ${ }^{1}$ Alison Bunce, ${ }^{2}$ Elaine Rae, ${ }^{3}$ Linda McEnhill1 ${ }^{1}$ The Prince \& Princess of Wales Hospice, Glasgow, Scotland; ${ }^{2}$ Ardgowan Hospice, Greenock, Scotland; ${ }^{3}$ St. Vincent's Hospice, Nr Johnstone, Scotland

10.1136/bmjspcare-2011-000105.12

A new and innovative 5 year lottery funded service which involves collaborative working and shared policies across three independent Hospices in NHS Greater Glasgow and Clyde. It offers information, support and help to families with children and young people aged 2-18 years who are facing challenging times in coping with life limiting illness and bereavement, recognising that dealing with grief at an early age can prevent worsening emotional and mental health issues in later life. (Every Child Matters 2004).

In the first year we successfully ran 2 groups; 5 teenagers then 5 children and worked individually with 47 others. They were offered a choice of counselling, play therapy or a mixture of both, at school, home or Hospice and were seen one to one using adapted models of established intervention (Winston's Wish and Solution Focused Care) or within a small age appropriate group. They were encouraged to talk about their feelings through interaction, play or arts and crafts.

We have established training, trained volunteers and plan to train teachers to assist in setting up groups supporting bereaved young people.

Results Initial evaluation of our teenage group identified that the young people appreciated being treated as equals, and the programme was adapted to extend the sessions following their feedback. We identified that getting children and young people together helped reduce the sense of isolation and helped them talk and learn about the grief process. The hospices have also agreed to extend bereavement support to other family members.

Future plans A third group has just been completed and a fourth planned. We have also begun researching the needs of very young bereaved children aged $2-5$ years highlighting the needs of this age group and the lack of awareness and training within nurseries. A website is to be established. 